\title{
Improving Multicast Stability in Mobile Multicast Scheme Using Motion Prediction`
}

\author{
Qian $\mathrm{Wu}^{1}$, Jianping $\mathrm{Wu}^{1}$, and Mingwei $\mathrm{Xu}^{2}$ \\ ${ }^{1}$ Department of Computer Science and Technology, Tsinghua University, \\ Beijing 100084, China \\ \{wuqian, jianping\}@cernet.edu.cn \\ ${ }^{2}$ Department of Computer Science and Technology, Tsinghua University, \\ Beijing 100084, China \\ xmw@csnet1.cs.tsinghua.edu.cn
}

\begin{abstract}
Stability is an important issue in multicast, especially in mobile environment where joining and leaving behaviors occur much more frequently. In this paper, we propose a scheme to improve the multicast stability by the use of motion prediction. The mobile node (MN) predicts the staying time before entering the new network, if the time is long enough, it will ask the new network to join the multicast tree as usual. Otherwise, the new network should create a tunnel to the multicast agent of $\mathrm{MN}$ to receive multicast packets. Considering that networks usually have different power range, the staying time is not predicted directly, and the Average Staying Time is used instead. The prediction algorithm is effective but practical which requires little calculation time and memory size. The simulation results show that the proposed scheme can improve the stability of multicast tree remarkably while bring much smaller cost.
\end{abstract}

\section{Introduction}

Mobile communication is playing an increasing role in our lives. Nowadays the most widely used mobility pattern is host mobility, often called terminal mobility, and this kind of mobile users always expect similar applications to static ones. Providing multicast in mobile networks is inspirited because IP multicast can provide many attractive applications, such as video conferencing, multiplayer games, etc, and bring the merit of efficient multi-destinations delivery which can save network bandwidth and release the burden of replications from the source.

In mobile environment, multicast must deal with not only dynamic group membership but also dynamic member location. But current multicast protocols are developed implicitly for static members and do not consider the extra requirements to support mobile nodes. Every time a member changes its location, keeping track of it and reconstructing the multicast tree will cause extreme overhead, while leaving the multicast tree unchanged will result in inefficient

\footnotetext{
* This work is supported by the Natural Science Foundation of China (No 60373010 and No 90604024), and National 973 Project Fund of China (No 2003CB314801).
} 
sometimes incorrect delivery path. Thus, providing multicast support for mobile nodes is challenging and faces with many new problems [2, 3, such as routing inefficiency, multicast group and tree maintenance overhead, handoff latency, packets loss and multicast tree stability.

Stability is an important issue in multicast, especially in mobile environment where joining and leaving behaviors occur much more frequently. Former study [6] shows that when the number of group members is not bigger than the $1 / 3$ of network size, the multicast tree would be an unstable one. Wu et al [7] find that the stability problem is more serious in mobile environment and it is mainly dominated by three elements, namely the ratio of the number of mobile nodes and network size, mobility model and the mobile multicast scheme.

In this paper we introduce a technique to improve the stability of multicast tree by the use of motion prediction, and apply it on the FHMM (Mobile Multicast with Fast Handoff and Hierarchical Architecture) [1 mobile multicast scheme. FHMM scheme has many advantages such as low packet loss rate, high multicast packet delivery efficiency and little multicast maintenance overhead. But it can be still improved in the aspect of multicast tree stability. The new scheme proposed in this paper is called P-FHMM. It uses a practical motion prediction algorithm to predict the staying time of $\mathrm{MN}$ in the new network. If the expected staying time is long enough, this network will be asked to join the multicast group as same as in FHMM. Otherwise, this network should only create a tunnel to the Multicast Agent (MA) of MN and use this tunnel to receive multicast packets. Simulation results show that, the stability of multicast tree can be obviously improved while its expense - the efficiency - will only decrease a little. While building tunnel will cause some additional handoff latency, the simulation results show that the packet loss percentage of P-FHMM scheme is still very small and within the acceptable range.

In section 2 we briefly introduce some mobile multicast schemes and the stability issue of multicast tree. Section 3 describes P-FHMM scheme and the motion prediction algorithm in detail. Section 4 presents our experiments using OMNet $++[11$ and results analysis. Finally, we conclude this paper.

\section{Background and Related Works}

\subsection{Mobile Multicast Schemes}

Mobile IP\&IPv6 5,4] provide two basic approaches to support mobile multicast, i.e., bi-directional tunneling (BT) and remote subscription (RS). Most of the other proposed mobile multicast solutions are based on them [2,3].

In BT approach, MN subscribes to multicast group through its home agent (HA), and uses the bi-directional tunnel between them to receive multicast packets. In this manner, there is no need to update multicast group state and multicast tree after MN's handoff, so there is no multicast maintenance overhead. But the multicast delivery path of $\mathrm{MN}$ is far from optimal, and routing inefficiency and bandwidth wasting become the main drawbacks. 
In RS approach, MN always re-subscribes to the multicast group when it changes the attachment to a new access network. It maintains most of the merits of multicast mechanism especially the high packet delivery efficiency, and people pay more attentions to it and propose many mobile multicast solutions based on it. But the main problem of RS and RS-based approaches is the overhead of maintaining the multicast delivery tree because joining and leaving behaviors occur much more frequently in mobile networks.

FHMM scheme [1] is based on RS approach and solves its main drawbacks. It introduces two kinds of agents: Multicast Agent (MA) and Domain Multicast Agent (DMA). MA is the extended Access Router (AR) responsible for providing multicast service to MNs in the access network. DMA is a multicast enabled router which manages multicast within its domain. Through the use of these two kinds of agents, FHMM achieves a hierarchical mobile multicast architecture which can shield the local movement from outside, efficiently reduce the change of inter-domain multicast delivery tree and decrease the multicast protocol overhead related to handoff. In addition, FHMM introduces a fast multicast handoff technology through which the AR of expected foreign network (called as $E A R$ ) can be notified to join the dedicated multicast group in advance, which helps MN to receive multicast packets more quickly after the handoff. Then both the handoff latency and the packet loss rate can be reduced.

But FHMM scheme does not solve the stability problem of intra-domain multicast tree. When the number of group member is not very big and the move speed of MN is fast, it will happen that the MN moves out of the range of the newly visited network just after this network having joined the multicast tree, or sometimes the MN may move to other networks before this network finishing joining the multicast tree. In this situation, the intra-domain tree will be caused to change frequently. What's more, due to the overlapping of networks, its fast multicast handoff mechanism may cause several networks to join the multicast group at the same time which would result in more serious stability problem.

\subsection{Stability of Multicast Tree}

Mieghem et al [6] studies how the number of links in a multicast tree changes after one multicast member leaves the group. For a multicast group with $m$ different members distributed in the graph containing $N$ nodes, the average number of link changes is denoted by $E\left[\Delta_{N}(m)\right]$, and when the expression $E\left[\Delta_{N}(m)\right] \leq 1$ is satisfied, the author claims the multicast tree would be a stable one.

Wu et al [7] studies the stability of IP multicast tree in mobile environment. In its expanded definition of stability, the link change of the multicast tree can be caused by three kinds of multicast group membership updates, namely join, leave and leave-join event. The author calls the value $E\left[\Delta_{N}(m)\right]$ as Stability Factor. The simulation results show that stability problem is more serious in mobile environment and the Stability Factor is mainly dominated by three elements. The first is the ratio of $m / N$ which reflects the density of mobile nodes in the network and the probability of MN moving to a network which already has MNs in its range. The second is the mobility model which effects how these mobile nodes 
are distributed in the network, and the aggregative degree of them. The third is the mobile multicast scheme which influences the way of updating multicast tree when a position change event happens. Although the effect of speed of MN and the power range of AR on the Stability Factor remains slight, both of them will cause the multicast tree to update more frequently.

\section{Proposed Scheme}

\subsection{Overview}

FHMM can reduce the change of inter-domain multicast tree, but the multicast tree inside the domain remains unimproved. And due to the overlapping of networks, its fast multicast handoff mechanism may cause more than one networks to join the multicast group at the same time which would result in more serious stability problem. Especially when the number of group members is not very big and the move speed of mobile nodes is fast, it will cause more transitory or sometimes unuseful joining actions, and then aggravate the stability problem.

In this paper, we propose P-FHMM scheme to improve the multicast stability of FHMM by the use of motion prediction. Every time the MN detects the connection to a new access network, it predicts the staying time in this network. If the expected staying time is long enough, this network will be asked to join the multicast group as same as in FHMM. Otherwise, this network should only create a tunnel to the MA of MN and use this tunnel to receive multicast packets. By the use of motion prediction, the short-term and unnecessary joining group action can be eliminated and the stability can be improved. What's more, P-FHMM only makes few modifications on FHMM and achieves visible performance.

P-FHMM can use any motion prediction algorithm to predict staying time. Here we use the Adaptive Response Rate Single Exponential Smoothing (ARRSES) 8] method. It is a simple but effective algorithm with little calculation and memory requirements. It is practical in mobile environment since the capability of mobile device is always limited than static one. What's more, in view that network may have different power range, we do not predict staying time directly. We use ARRSES algorithm to predict the average staying time in the new network instead, and then the expected staying time can be worked out.

\subsection{Operation Details}

\section{Modifications of FHMM}

As mentioned in [1], the operation of FHMM include two main procedures, the first is multicast group joining procedure and the second is handoff procedure. The multicast group joining procedure of P-FHMM is the same as FHMM, while the fast multicast handoff procedure has some modifications.

The first modification is adding a " $T$ " flag in the multicast group option in fast multicast handoff procedure. If the "T" flag is set, it means the prediction result 
made by MN is using tunnel. Otherwise, the result is joining multicast group as usual. Through the exchange of messages carrying multicast group option, the EAR in the new network can be informed with the prediction result.

The second modification is adding a new message named FNABack (Fast Neighbor Advertisement Acknowledge). In FHMM scheme, as soon as the MN is handoff to a new network, it sends a FNA (Fast Neighbor Advertisement) message to announce its attachment. We add this new FNABack message to acknowledge FNA message, and thus MN can know the ultimate handoff decision of EAR in this new network and then change its own handoff decision and modify its MA information correspondingly. It is used to ensure efficiency. For example, if the "T" flag is set but the EAR has already been on the multicast tree, there is no need to create a tunnel transferring the same multicast packets.

The last modification is adding the power range information of EAR in PrRtAdv (Proxy Router Advertisement) message. In FHMM scheme, the PrRtAdv message is used to tell the MN the address information of EAR. After this modification, the $\mathrm{MN}$ can also know the power range of EAR and this information is needed in our prediction algorithm.

\section{Handoff procedure}

This section introduces the multicast handoff procedure of P-FHMM in detail, especially the difference with FHMM scheme.

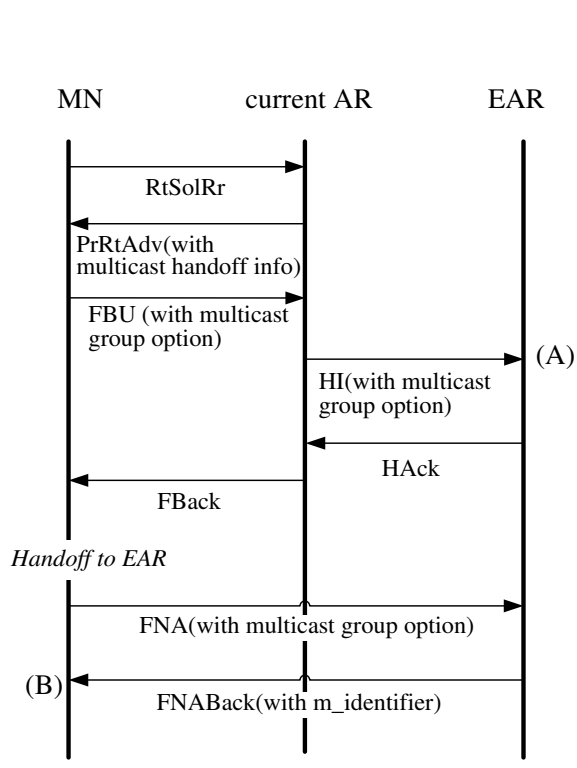

(a) Messages Exchanged

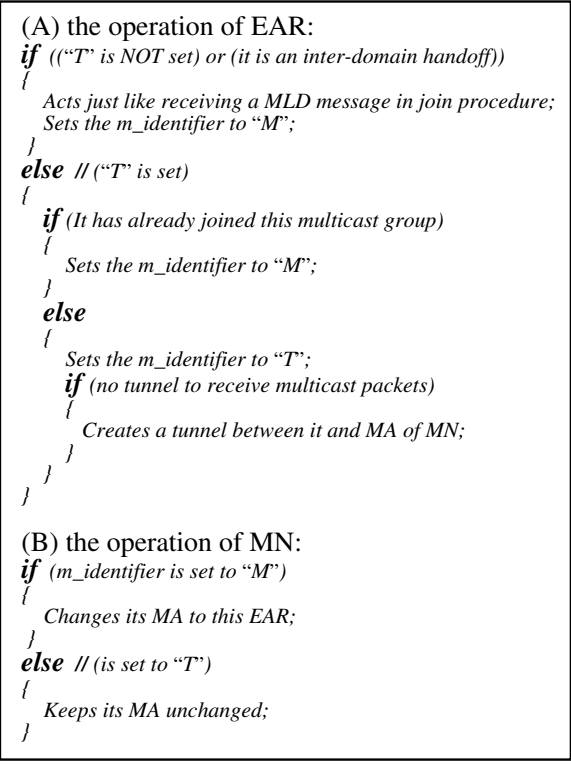

(b) Judgments and Operations

Fig. 1. Handoff Procedure of P-FHMM 
Multicast handoff in P-FHMM is still fast and efficient. Fig 1 shows the handoff . As we can see, the main operations of multicast handoff procedure change a little. Fig 1(a) illustrates the messages exchanged in handoff procedure. They are the same as FHMM scheme except the new FNABack message used in the final stage. Fig 1(b) shows the detailed judgments and operations of MN and EAR.

When MN discovers the availability of a new access network by some linkspecific methods while still connected to its current network, it starts fast multicast handoff procedure. By the information of EAR's power range gained in PrRtAdv message, MN predicts the staying time in the new network. If this expected staying time does not reach the pre-assigned value, the prediction result is using tunnel, and the "T" flag is set. Otherwise the "T" flag should be cleared. Then through the use of FBU (Fast Binding Update) and HI (Handover Initiate) messages, MN can transfer multicast group option carrying "T" flag information to EAR which would motivate EAR to determine the ultimate handoff decision. EAR will make the ultimate handoff decision to joining multicast tree in three situations: the "T" flag in multicast group option is not set which means the expected staying time is long enough, or EAR itself has already joined this multicast tree which means there is no need to create a tunnel anymore, or the handoff is an inter-domain one and EAR should join the multicast tree in order to keep the hierarchical architecture. Otherwise the ultimate handoff decision is set to using tunnel and EAR should create a tunnel to the MA of MN.

When the $\mathrm{MN}$ is disconnected from the previous network and moves to the new access network, it uses FNA message to quickly announce its attachment to EAR. For robustness, FNA message also includes the multicast group option. EAR acknowledges it with FNABack message which carries its ultimate handoff decision. If this ultimate handoff decision is joining multicast tree, MN should changes its MA to this EAR, otherwise, its MA information remains unchanged.

\subsection{Motion Prediction Algorithm}

Because the power range of networks may be different and it is one of the decisive factors of predicting staying time of $\mathrm{MN}$ in the network, it will not be precise if the prediction algorithm does not consider the factor of power range. In our motion prediction algorithm we do not predict staying time directly, instead we predict the average staying time and then calculate the expected staying time.

Definition 1. For the ith $(i \geq 1)$ access network a mobile node visited, we call the value $\eta_{i}=T_{i} / R_{i}$ as Average Staying Time of mobile node in this network, where the $R_{i}$ is the power range of this network and $T_{i}$ is the staying time.

To some extent, the average staying time represents the average move speed of MN in the network, and our motion prediction algorithm runs on it. This is because that for more accurate prediction result, we should use Globe Positioning System (GPS) or something else with the same effect to get the exact move speed, move direction and location of MN and also the location of MA, and then we can predict the staying time more precisely. But the GPS is not always 
available and the capability of mobile device is usually limited than static one, the burdensome calculation of prediction is not always acceptable. What's more, the mobility of MN has the property of randomness, even if MN knows all the precise information and has enough calculation capability, the exact value can not always be predicted.

Facing with the fact that a mobile user usually travels with a destination in mind, and the change of mobile node's velocity within a short time is limited due to physical restrictions, we predict the future average move speed, i.e. average staying time, of MN correlating to the previous and current ones.

The prediction method we use is the Adaptive Response Rate Single Exponential Smoothing (ARRSES) algorithm introduced by Trigg and Leach [8]. It is a very popular and effective forecasting method with the merit of easy to use, very little calculation and memory requirements, the ability of automatic adjusting the predicting parameter to forecasting error. It is a variation of Exponential Smoothing method and can continually adjust the Smoothing Parameter $\alpha_{i}$.

The expected average staying time $\left(\tilde{\eta}_{n+1}\right)$ is calculated as follows:

$$
\tilde{\eta}_{n+1}=\alpha_{n} \times \eta_{n}+\left(1-\alpha_{n}\right) \times \tilde{\eta}_{n}
$$

where the $\tilde{\eta}_{n+1}$ and $\tilde{\eta}_{n}$ are the prediction value of $n+1$ and $n$ network, $\eta_{n}$ is the real value of $n$ network, and $\alpha_{n}$ is smoothing parameter to predict the value in $n+1$ network and $0 \leq \alpha_{n} \leq 1 . \alpha_{n}$ is calculated by following steps:

$$
\begin{gathered}
e_{n}=\eta_{n}-\tilde{\eta}_{n} \\
E_{n}=\beta e_{n}+(1-\beta) E_{n-1} \\
M_{n}=\beta\left|e_{n}\right|+(1-\beta) M_{n-1} \\
\alpha_{n}=\left|E_{n} / M_{n}\right|
\end{gathered}
$$

where the parameter $\beta$ is usually set at 0.1 or 0.2 .

Then the expected staying time can be worked out by $\tilde{T}_{n+1}=\tilde{\eta}_{n+1} \times R_{n+1}$, and if $\tilde{T}_{n+1}$ is not smaller than the threshold value $T_{t h}$, the handoff decision is joining multicast tree and the "T" flag should be cleared, otherwise the handoff decision is using tunnel and the "T" flag should be set.

\section{Performance Evaluation}

\subsection{Network Model and Methodology}

The simulation is built on OMNET $++[11$, a discrete event simulator. We compare the stability of P-FHMM with FHMM in terms of stability factor and the multicast tree change frequency. We also investigate the main costs of improving stability - the packet delivery efficiency and the packet lost rate. 
The topology in our simulation is a $10 \times 10$ mesh network in which each node acts as a multicast router of local network and also an MA for MN. There are 4 DMA routers in the topology, and each DMA is responsible for $25(5 \times 5)$ MAs. The power range is $71 \mathrm{~m}$, and the distance between two nearby MAs is $100 \mathrm{~m}$.

For simplicity, there is one multicast group with one data source. The source generates multicast packet with the length of 300 bytes at intervals of $20 \mathrm{~ms}$ to simulate the multicast conference application.

In our simulation, multicast group members are all mobile nodes, and the update of multicast tree is absolutely caused by the position change events of mobile members. The number of mobile nodes varies from 5 to 30. Originally, mobile nodes are randomly located at the mesh network.

For all the performances investigated, we check them both in Random Waypoint [9] (RPW for short) and Gauss-Markov [10] (GM for short) mobility model. In RPW model, the MN at first chooses a random destination in the simulation area and a speed. It then travels toward this new destination. Upon arrival, it pauses for a random period before starting the process again. In our simulation we set the pause time to 0 to evaluate the performance in most random condition. The speed is uniformly chosen between 0 and $30 \mathrm{~m} / \mathrm{s}$.

While in GM mobility model, MN's new speed and direction are correlated to its formal speed and direction and their mean values through the simulation. This model uses a tuning parameter, $\alpha$, where $0 \leq \alpha \leq 1$, to vary the randomness. The smaller the $\alpha$, the greater the randomness is. We choose the value of $\alpha$ to 0.8 to simulate the move with high historical character, and the mean value of speed is fixes to $30 \mathrm{~m} / \mathrm{s}$ which reflects the MN with high speed.

We run each simulation for 500 seconds.

\subsection{Simulation Results}

Fig 2 to 8 are the simulation results. To investigate the P-FHMM scheme, we examine three different staying time thresholds, namely 3, 5 and 10 seconds.

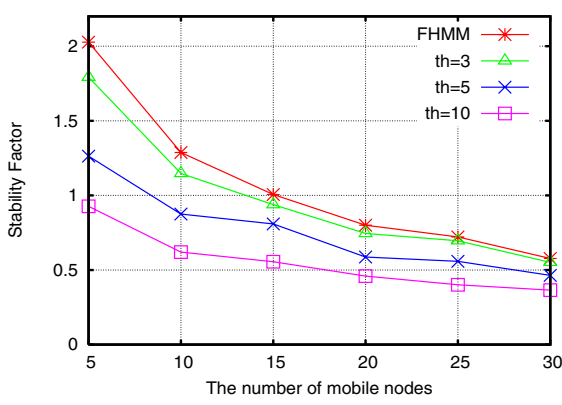

(a) Comparing under RWP Model

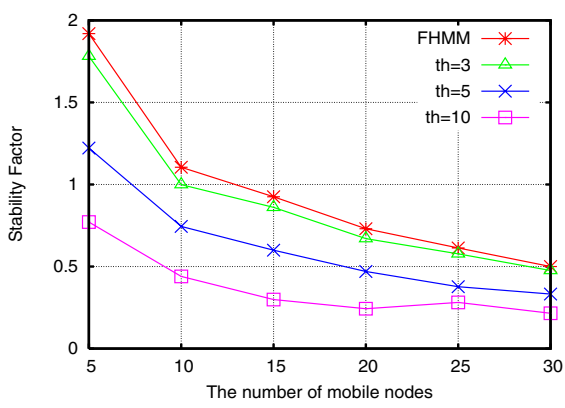

(b) Comparing under GM Model

Fig. 2. Comparison of Stability Factor 


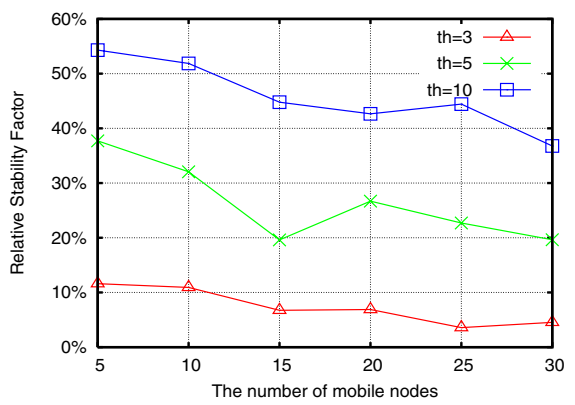

(a) under RWP Model

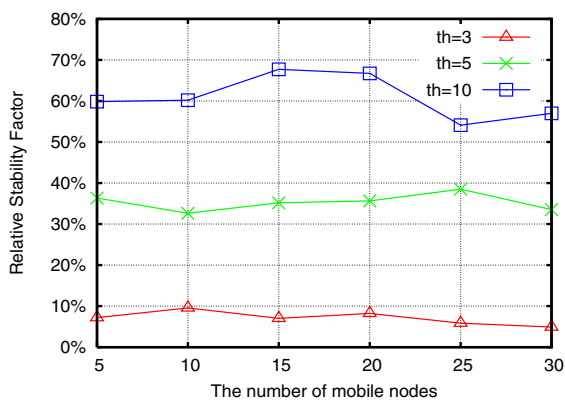

(b) under GM Model

Fig. 3. Relatively Reduced Value of Stability Factor

Fig 2 compares the stability factor. Fig [3 is the relatively reduced value of stability factor of P-FHMM which reflects the improvement on FHMM scheme. From the four pictures in these two figures we can see: 1) through the use of motion prediction, the stability factor of mobile multicast scheme can be improved; 2) the longer the staying time threshold of joining multicast tree, the smaller the stability factor which means the more stable of the multicast tree; 3) when the staying time threshold is about 5 seconds, the stability can be improved by about $30 \%$ and the stability factor is nearly all below 1, the stable regime defined in [6] and [7].

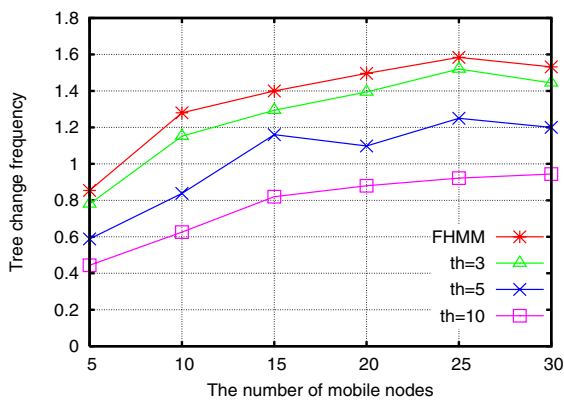

(a) Comparing under RWP Model

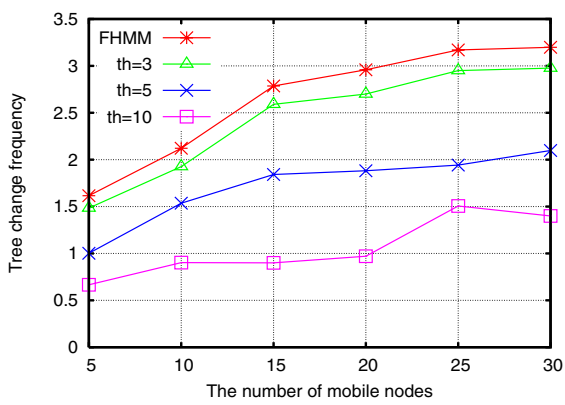

(b) Comparing under GM Model

Fig. 4. Comparison of Multicast Tree Change Frequency

Fig 4 and 5 illustrate the simulation results of multicast tree change frequency. Fig 4 compares the absolute value and fig 5 is the relatively reduced value. From the figures we can conclude that through the using of motion prediction, the frequency of modifying multicast tree can be reduced remarkably. And the longer the staying time threshold of joining multicast tree will result in less modification frequency and better improvement of stability.

What's more, we find that fig 5 is very similar to fig 3 . Stability factor and multicast tree change frequency are two main elements of investigating multicast 


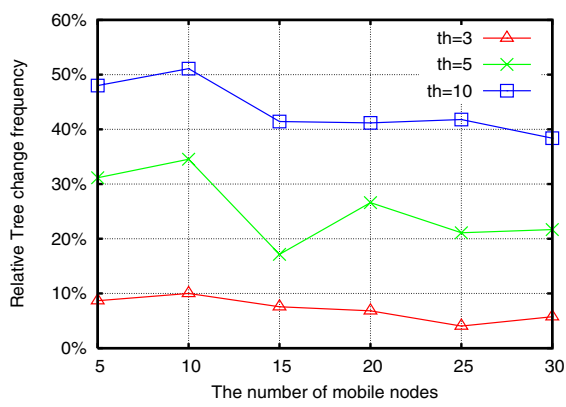

(a) under RWP Model

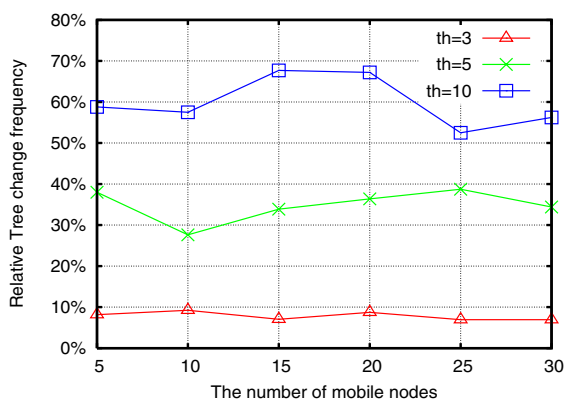

(b) under GM Model

Fig. 5. Relatively Reduced Value of Multicast Tree Change Frequency

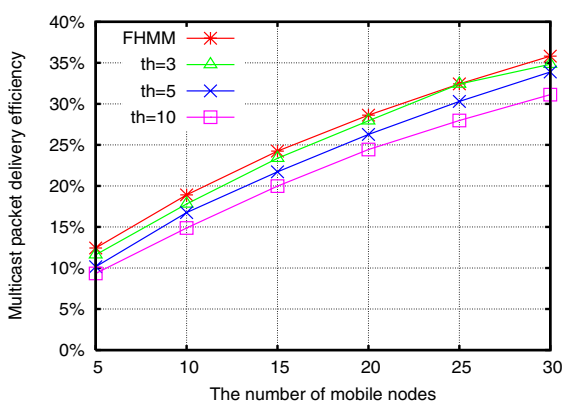

(a) Comparing under RWP Model

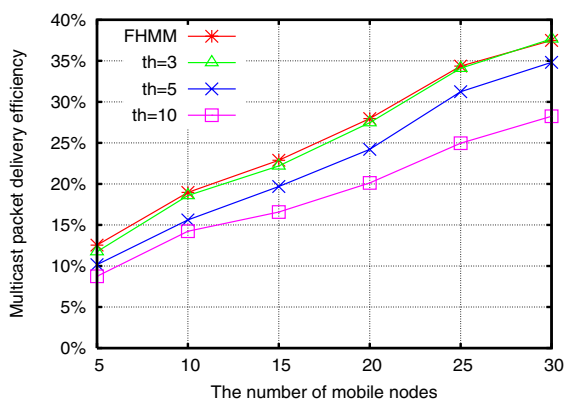

(b) Comparing under GM Model

Fig. 6. Comparison of Multicast Packet Delivery Efficiency

stability. The similarity of these two figures reflects that P-FHMM scheme can equally improve both of these two stability elements, which verifies its effectivity.

For the use of tunnel, the main cost of improving stability is the packet delivery efficiency. Fig [6] and 7 compare the multicast packet delivery efficiency of PFHMM and FHMM. Fig [6 is the comparison of absolute value and fig 7 is the relatively reduced value. From these two figures we can have some conclusions. Firstly, improving stability will certainly reduce the packet delivery efficiency. This is because the pure multicast is the most efficient mechanism and the use of tunnel will decrease a part of multicast to unicast. Secondly, the longer the staying time threshold is, the more inefficient the mobile multicast scheme will be. This is because that longer threshold will result in more tunnels to be used. Thirdly, and also the most important, as shown in fig 7 the decreasing of efficiency is much smaller (nearly the half of) than the improvement of stability illustrated in fig 3 and fig 5 . Let's look at line of threshold 5 seconds for example. The improvement of stability of the proposed P-FHMM scheme in fig 3 and fig 5 is around $30 \%$, while the cost, namely decreased efficiency shown in fig 7, is just around $15 \%$. This result means P-FHMM scheme uses little cost to gain more significant improvement, and further verifies its effectivity. 


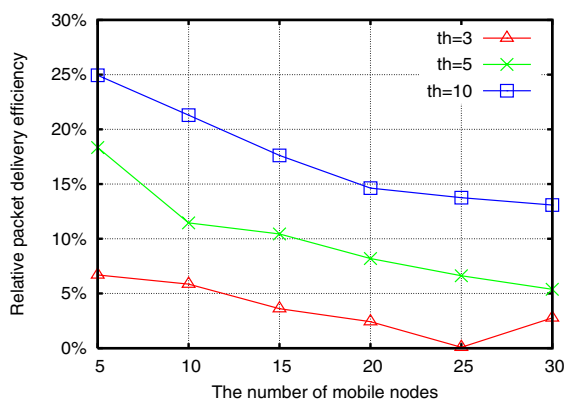

(a) under RWP Model

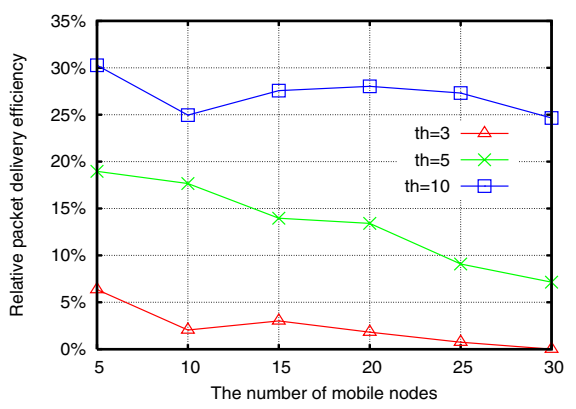

(b) under GM Model

Fig. 7. Relatively Reduced Value of Multicast Packet Delivery Efficiency

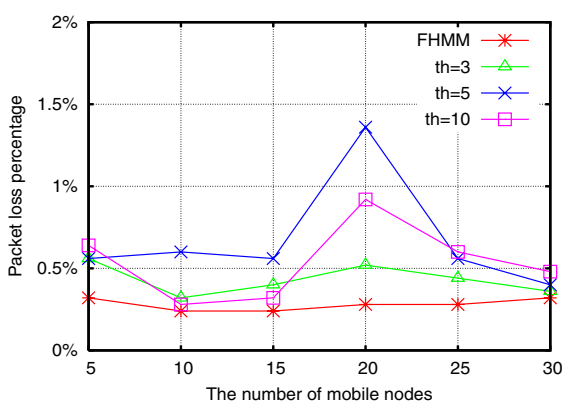

(a) Comparing under RWP Model

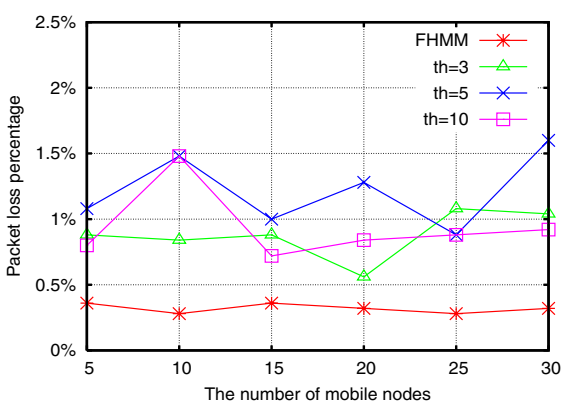

(b) Comparing under GM Model

Fig. 8. Comparison of Multicast Packet Lost Percentage

Fig 8 is the comparison of multicast packet lost percentage. Obviously the FHMM scheme achieves the best performance. For the need of building the tunnel, P-FHMM introduces new handoff latency and this would cause more packets to be lost. But the packet lost percentage keeps relatively uninfluenced with the staying time threshold, and although more packets have been lost, the packet lost percentage of P-FHMM is still around $0.5 \%$ under RPW model and $1 \%$ under GM model. These results are still acceptable for multicast applications.

\section{Conclusion and Future Work}

In this paper, we propose a scheme (P-FHMM) to improve the multicast stability of FHMM by the use of motion prediction. In the multicast handoff procedure of P-FHMM scheme, MN predicts its staying time in the new network at first. If the expected staying time is long enough, it will ask the new network to join the multicast tree as usual. Otherwise, the new network will only be asked to create a tunnel to the multicast agent of $\mathrm{MN}$ and use this tunnel to receive multicast packets. To achieve this, the P-FHMM scheme makes some modifications on 
FHMM. But the main multicast handoff procedure remains unchanged and is still efficient, and the prediction algorithm introduced by P-FHMM is effective but practical which requires little calculation time and memory size. We set up simulation to evaluate the performance. From the abundant simulation results we can see that P-FHMM scheme is effective in improving the stability of multicast tree, and the improvement is remarkable. We investigate the stability in terms of stability factor and the multicast tree modification frequency, and in both sides P-FHMM can obtain good performance and the improved results are similar. In the mean time, the main cost, namely decreasing of efficiency, is much smaller than the improvement. And the other cost, the packet lost percentage is still within an acceptable range.

\section{References}

1. Qian, Wu. (ed.): An Agent-Based Scheme for Efficient Multicast Application in Mobile Networks. ISCIS 2005, LNCS 3733. (2005) 23-32

2. I, Romdhani., M, Kellil., H-Y, Lach., A, Bouabdallah., H, Bettahar.: IP Mobile Multicast: Challenges and Solutions. IEEE Communications Surveys \& Tutorials, vol.6, no.1. (2004) 18-41

3. Hrishikesh, Gossain., Carlos, De.M.Cordeiro., Dharma, P.Agrawal.: Multicast: Wired to Wireless. IEEE Communications Magazine, vol.40, no.6. (2002) 116-123

4. D, Johnson., C, Perkins., J, Arkko.: Mobility Support in IPv6. RFC 3775, June (2004)

5. C, Perkins. (ed.): IP Mobility Support for IPv4. RFC 3344, August (2002)

6. P, V.Mieghem., M, Janic.: Stability of a Multicast Tree. IEEE INFOCOM 2002, Vol.2. Anchorage, Alaska (USA) (2002)1133-1142

7. Qian Wu. (ed.): A Simulation Study to Investigate the Impact of Mobility on Stability of IP Multicast Tree. MSN 2005, LNCS 3794. (2005) 836-845

8. D. W. Trigg., D. H. Leach.: Exponential Smoothing with an Adaptive Response Rate, Operational Research Quarterly, vol. 18. (1967) 53-59

9. D, Johnson., D, Maltz.: Dynamic source routing in ad hoc wireless networks. In T. Imelinsky and H. Korth, editors, Mobile Computing. (1996) 153-181.

10. B, Liang., Z, Haas.: Predictive distance-based mobility management for PCS networks. Proceedings of the Joint Conference of the IEEE Computer and Communications Societies (INFOCOM). (1999) 1377-1384

11. Omnet++ Community Site. http://www.omnetpp.org 